\title{
A New Strategy for the Synthesis of Nonsymmetrical 3,3'-(Aryl/alkyl-methylene) bis-2-hydroxy-1,4-naphthoquinones and Their Cytotoxic Effects in PC3 Prostate Cancer Cells
}

\author{
Ruan Carlos B. Ribeiro, ${ }^{\#, a}$ Paula Priscilla de Freitas, ${ }^{\#, b}$ Caroline S. Moreira, ${ }^{a}$ \\ Leonardo G. C. de Moraes, ${ }^{a}$ Matheus G. de Moraes, ${ }^{a}$ Fernando C. da Silva, ${ }^{\odot a}$ \\ David R. Rocha, ${ }^{*, a}$ Etel R. P. Gimba ${ }^{*, a, c}$ and Vitor F. Ferreira ${ }^{*, d}$ \\ anstituto de Química, Universidade Federal Fluminense, Campus do Valonguinho, \\ 24020-150 Niterói-RJ, Brazil \\ ${ }^{b}$ Coordenação de Pesquisa, Programa de Oncobiologia Celular e Molecular, \\ Instituto Nacional de Câncer, 20231-050 Rio de Janeiro-RJ, Brazil \\ 'Departamento de Tecnologia Farmacêutica, Faculdade de Farmácia, \\ Universidade Federal Fluminense, Santa Rosa, 24241-002 Niterói-RJ, Brazil \\ ${ }^{d}$ Departamento de Ciências da Natureza (RCN), Instituto de Humanidades e Saúde, \\ Universidade Federal Fluminense, 28880-000 Rio de Janeiro-RJ, Brazil
}

\begin{abstract}
A novel method for the synthesis of nonsymmetrical 3,3'-(aryl/alkyl-methylene)bis-2-hydroxy1,4-naphthoquinones was developed by using the Mannich adduct of naphthoquinone and the reaction with another moiety of 2-hydroxy-1,4-naphthoquinone. This novel method produces for the first time nonsymmetrical 3,3'-(aryl/alkyl-methylene)bis-2-hydroxy-1,4-naphthoquinones. In a preliminary study, these compounds $(\mathbf{1 5 c}, \mathbf{1 5 f}$ and $\mathbf{1 5 h})$ were evaluated regarding their effect over the viability of PC3 metastatic prostate cancer cell using 3-(4,5-dimethylthiazol-2-yl)2,5-diphenyltetrazolium bromide (MTT) assays at $100 \mu \mathrm{M}$. Three of these compounds presented relevant cytotoxic effects at $72 \mathrm{~h}$ posttreatment.
\end{abstract}

Keywords: 3,3'-arylmethylenes, 3,3'-alkylmethylenes, nonsymmetrical bis(2-hydroxynaphthalene-1,4-dione), lawsone, Mannich adduct, quinone methide

\section{Introduction}

Nature is capable of producing many substances, which for centuries have been used by mankind to treat many diseases. Quinones are among the most common natural compounds. ${ }^{1,2}$ Evolutionary biology has shown that some insects and plants use these compounds as chemical defense, but most of them are used for biochemical functions in specific endogenous oxidative processes, such as photosynthesis, electron transfer, and blood coagulation. These biological activities are mainly related to the redox cycle involving anions, dianions, superoxide anions,

*e-mail: davidrrocha@uol.com.br; etelgimba@id.uff.br;

vitorferreira@id.uff.br

\#These authors equally contributed on performing the experiments described in this article.

*These authors equally contributed to this work and are co-senior authors. and semiquinone radicals in situ, all of which accelerate intracellular hypoxia conditions and then promote the synthesis of reactive oxygen species (ROS). ${ }^{3-8}$ Indeed, it has been proven that small molecules that induce the formation ROS can selectively target overexpressed enzymes in cancer cells. ${ }^{9}$

The largest group of compounds of the quinone class is the naphthoquinone group. These compounds are widely distributed in plant and animal kingdoms, performing several roles. Several natural and synthetic compounds containing the naphthoquinone nucleus became drugs in the pharmaceutical market, including lapachol (1), atovaquone (2), ${ }^{10}$ buparvaquone (3) ${ }^{11}$ and others that are being screened in clinical trials, such as $\beta$-lapachone $(\mathbf{4}),{ }^{12}$ as shown in Figure 1.

The structural diversity of naphthoquinones has been explored as a strategy to discover new bioactive 

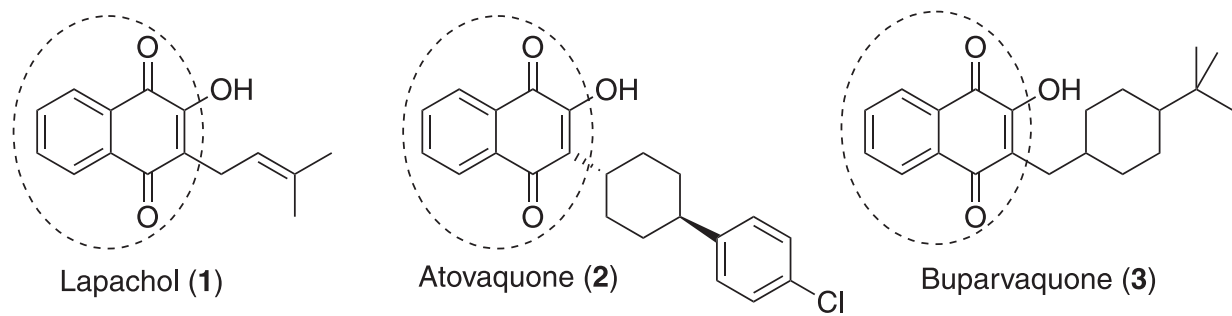

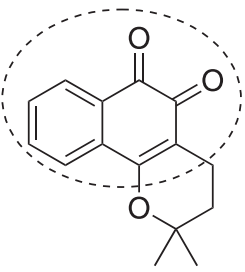

$\beta$-Lapachone (4)

Figure 1. Naphthoquinones used in the clinic or under clinical trials.

substances. One particular class of natural and synthetic symmetrical compounds having the fragment 2,5-diene2,6-diol with general structure (5, Figure 2) has demonstrated good biological activity in several pharmacological targets. ${ }^{13}$ Benzylidene bisphenols (6), ${ }^{14,15}$ benzylidene bisnaphthols (7), ${ }^{16}$ biscoumarins $(\mathbf{8})^{17,18}$ and 3,3'-(methylene)bis-2-hydroxy-1,4-naphthoquinones (9) are included in this class of compounds. The bestknown compound of this class is anticoagulant (9) (Figure 2), which has been used as a synthetic analogue instead of warfarin (Figure 2). It is noteworthy to mention that diphthiocol 11 (dicoumarol, Figure 2) is a natural 3,3'-arylmethylene isolated from root cultures of Impatiens balsamina. ${ }^{19}$

Regarding the series of 3,3'-(methylene)bis-2-hydroxy1,4-naphthoquinones (9, Figure 2), these compounds have been prepared by several methods, but their biological properties are poorly known. Tisseh and Bazgir ${ }^{20}$ developed an efficient and clean synthesis of these compounds in an aqueous solution of LiCl. Otherwise, de Oliveira et al. ${ }^{21}$ found that the same reaction in water under ultrasound irradiation produced these products in better yields. de Araújo et al. ${ }^{22}$ also prepared these compounds in high yields through the condensation of lawsone (1) with aromatic and aliphatic aldehydes under mild acidic catalysis of $\beta$-alanine/AcOH and assayed then against Leishmania amazonensis and Leishmania braziliensis promastigotes. Some of them showed good leishmanicidal activity without significant toxic effects. de Araújo et al..$^{22}$ found that some of these compounds showed promising leishmanicidal activity. In another study, Sharma et al. ${ }^{23}$ tested these types of compounds against Leishmania donovani promastigotes and on leishmanial deoxyribonucleic acid (DNA) topoisomerase I. Regarding the anticancer activity, there is one report published by Sadhukhan et $a l .{ }^{24}$ who proposed the synthesis of symmetrical 3,3'-(aryl/methylene)bis2-hydroxy-1,4-naphthoquinones as potential anticancer agents. However, no biological result against tumor cells is shown in this publication. Brahmachari ${ }^{25}$ synthesized a series of 3,3'-(aryl/alkyl-methylene) bis(2-hydroxynaphthalene-1,4-dione) derivatives and assayed them for pro-apoptotic activity in five distinct

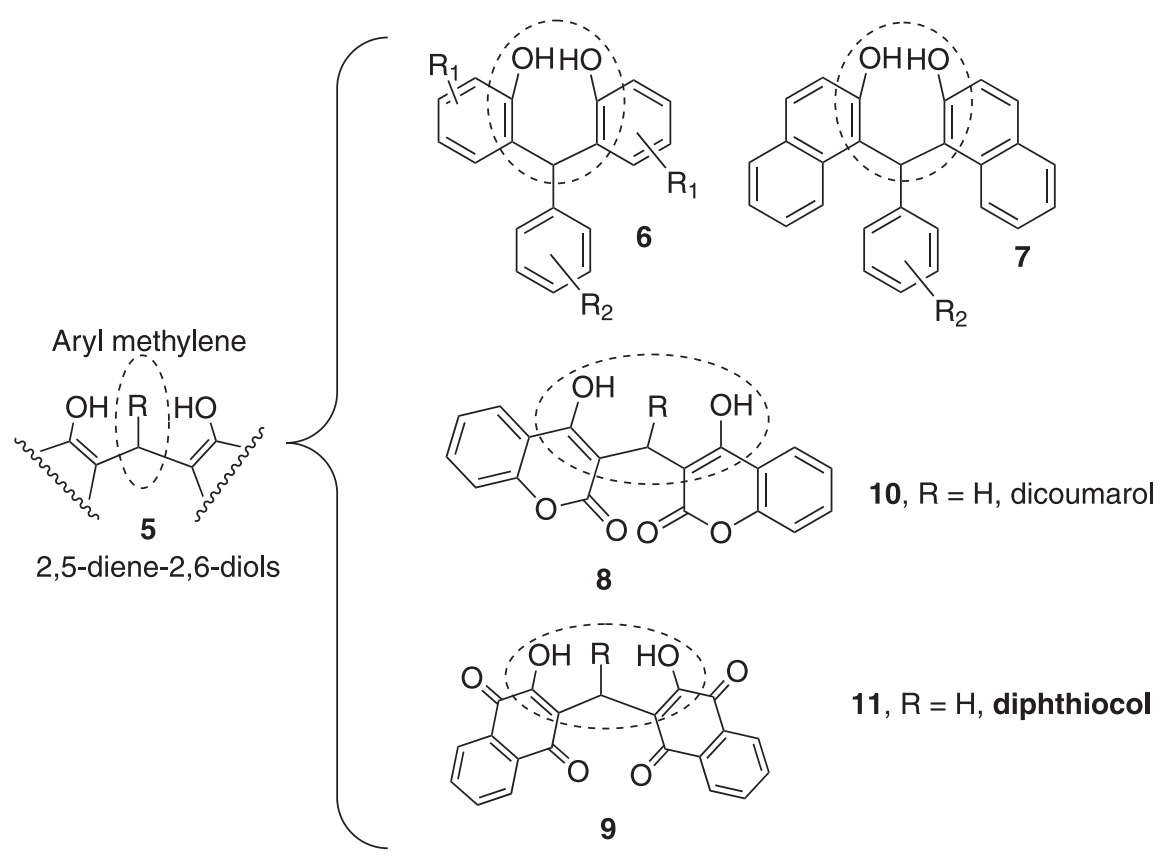

Figure 2. General structures of compounds 6-11 containing the 2,5-diene-2,6-diols moiety. 
cancer cell lines and found one compound effective in inducing apoptosis in human glioma cells (CCF-4) ${ }^{24}$

Although several methods for the synthesis of these derivatives have been reported, most of them suffer limitations regarding selectivity in forming nonsymmetrical 3,3'-(methylene)bis-2-hydroxy-1,4-naphthoquinones. In addition, data regarding the effect of these compounds in cancer cells are lacking.

It is noteworthy that there are several compounds containing only one naphthoquinonoid nucleus that have been shown to be active against prostate cancer cells. $\beta$-Lapachone analogs, ${ }^{26}$ poly(D,L)-lactide-co-glycolide (PLGA) microcapsules containing nor- $\beta$-lapachone ${ }^{27}$ plumbagin isolated from Plumbago zeylanica, ${ }^{28,29}$ shinkonin isolated Lithospermum erythrorhizon,,$^{30}$ and menadione hybrids triazoles ${ }^{31}$ are cytotoxic against PC3 or DU145 cancer cells by increasing intracellular ROS levels or inducing apoptotic cell death. Additionally, naphthoquinones with multimeric redox-active centers exhibit activities against highly proliferative neoplastic cells..$^{32,33}$

Thus, considering the increasing importance of these compounds (e.g., dicoumarol) and the reported reaction conditions for their synthesis, herein we describe our efforts toward the synthesis of nonsymmetrical 3,3'-(methylene) bis-2-hydroxy-1,4-naphthoquinones using two different naphthoquinone scaffolds. We then aimed to investigate the potential application of these compounds as putative anticancer agents by testing them in the PC 3 prostate cancer cell line as an experimental model.

\section{Results and Discussion}

The current literature has shown that the usual method to synthesize these bis-2,5-diene-2,6-diol compounds is the domino Knovenagel condensation of an enol with aldehydes, followed by a Michael reaction conducted with different acid catalysts, ${ }^{13}$ as shown in Figure 3. As mentioned before, one of the limitations in the preparation of these compounds (6-11) is that the naphthoquinones attached to the aryl-methylene are the same. Up to now, there are no efficient methods for the preparation of 3,3'-(methylene)bis-2-hydroxy-1,4-naphthoquinones with different naphthoquinones.
In this context, we developed a new two-step method for the synthesis of nonsymmetric 3,3'-(aryl/alkyl/methylene) bis-2-hydroxy-1,4-naphthoquinones with two different naphthoquinones, 2,5-dihydroxynaphthalene-1,4-dione and 2-dihydroxynaphthalene-1,4-dione, which were used as naphthoquinone scaffolds to prepare compound 15a-i (Scheme 1 and Figure 4). In this protocol, we used Mannich adducts (13a-i) that were easily prepared by a previously known method ${ }^{34-36}$ from 1.0 equivalent of lawsone (12), 1.1 equivalent of benzylamine and 1.1 equivalents of an aldehyde, dissolved in ethanol, kept under stirring at room temperature for $24 \mathrm{~h}$. Then, the mixture was filtered, and the solid was washed with water, and after drying under vacuum, the products (13a-i) were obtained as an orange or red solid in yields of $69-92 \%$ (Scheme 1). It is noteworthy that during this study Ghodsi et al. ${ }^{37}$ reported that the use of the Mannich reaction of lawsone with various aldehydes and $4 H-1,2,4$-triazol-4-amine gave unexpected symmetrical 3,3'-(arylmethylene)bis(2-hydroxynaphthalene-1,4-dione) derivatives.

For the reaction of the Mannich adducts (13a-i), we used the 2,5-dihydroxynaphthalene-1,4-dione (14) ${ }^{38}$ under acidic catalysis by acetic acid to furnish the nonsymmetrical 3,3'-(aryl/alkyl/methylene)bis2-hydroxy-1,4-naphthoquinones (15a-i) derivatives in low to moderate yields (Table 1). Depending on the purification method of the reaction mixture, the resulting yield can vary considerably, as shown in Table 1 . The chromatographic method systematically produced the compounds in better yields. All structures of Mannich adducts (13a-i) and 3,3'-(aryl/alkyl/methylene)bis2-hydroxy-1,4-naphthoquinones (15a-i) were confirmed by analysis of nuclear magnetic resonance (NMR) spectroscopy ${ }^{1} \mathrm{H}$ and ${ }^{13} \mathrm{C}$, infrared spectroscopy (IR), and high resolution mass spectrometry (HRMS). The Mannich adduct presented two characteristic signals in the form of a doublet corresponding to the two methylene hydrogens from benzylamine. All HRMS of final products 15a-i indicated that the structure has the correct molecular weight. However, they presented three singlet signals corresponding to the $\mathrm{C}-\mathrm{H}$ ( $\delta$ 6.23-6.05 ppm for 15i), confirming the incorporation of the second naphthoquinone moiety in the structure (see

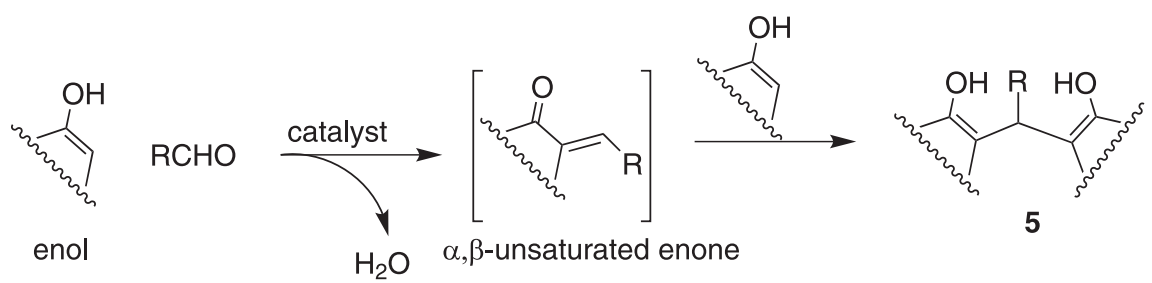

Figure 3. General synthetic routes for preparing compounds containing the 2,5-diene-2,6-diol moiety (5). 


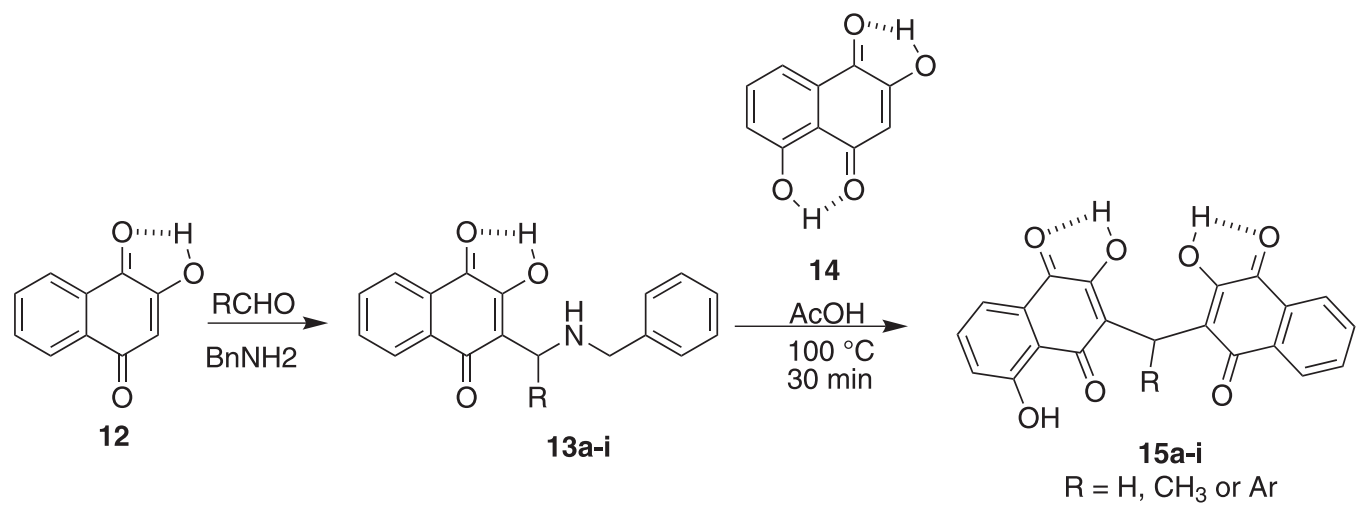

Scheme 1. Synthetic route used for the preparation of naphthoquinone 15a-i derivatives.

Table 1. Yields of synthesized 3,3'-(methylene)bis-2-hydroxy1,4-naphthoquinones 15a-i

\begin{tabular}{lccc}
\hline Compound & $\mathrm{R}$ & $\begin{array}{c}\text { Crystallization / } \\
\%\end{array}$ & $\begin{array}{c}\text { TLC } \\
\text { chromatography / \% }\end{array}$ \\
\hline $\mathbf{1 5 a}$ & $\mathrm{H}$ & 17 & 65 \\
$\mathbf{1 5 b}$ & $4-\mathrm{CH}_{3}-\mathrm{C}_{6} \mathrm{H}_{4}$ & 16 & 62 \\
$\mathbf{1 5 c}$ & $4-\mathrm{Br}_{-} \mathrm{C}_{6} \mathrm{H}_{4}$ & 23 & 44 \\
$\mathbf{1 5 d}$ & $4-\mathrm{NO}_{2}-\mathrm{C}_{6} \mathrm{H}_{4}$ & 47 & 55 \\
$\mathbf{1 5 e}$ & $\mathrm{CH}_{3}$ & 22 & 69 \\
$\mathbf{1 5 f}$ & $4-\mathrm{Cl}_{-}-\mathrm{C}_{6} \mathrm{H}_{4}$ & 51 & 59 \\
$\mathbf{1 5 g}$ & $4-\mathrm{F}-\mathrm{C}_{6} \mathrm{H}_{4}$ & 26 & 47 \\
$\mathbf{1 5 h}$ & $4-\mathrm{OCH}_{3}-\mathrm{C}_{6} \mathrm{H}_{4}$ & 20 & 41 \\
$\mathbf{1 5 i}$ & $\mathrm{C}_{6} \mathrm{H}_{5}$ & 56 & 61 \\
\hline
\end{tabular}

TLC: thin layer chromatography.

Supplementary Information (SI) section), but in the form of tautomers that are interconverted through the reallocation of a proton. The correlated spectroscopy (COSY) spectrum $\left({ }^{1} \mathrm{H} \times{ }^{1} \mathrm{H}\right.$ coupling $)$ of compound $\mathbf{1 5 i}$ showed no correlation for the three proton signals of $\mathrm{C}-\mathrm{H}$, confirming that they are signals of the tautomers. The methylation reaction of $\mathbf{1 5 i}$ with diazomethane yielded a compound that showed only a singlet signal for $\mathrm{C}-\mathrm{H}$ at $6.07 \mathrm{ppm}$ and three singlet signals for the methoxyls at 3.72, 3.74 and $3.76 \mathrm{ppm}$. Further NMR studies using different solvents at different temperatures have shown that the tautomeric balance was not significantly modified. As this tautomeric equilibrium is not observed in the symmetric adducts of lawsone (12), the origin of these tautomers is connected with the 2,5-dihydroxynaphthalene-1,4-dione moiety (14). It is reported in the literature that the tautomeric structure of 2-hydroxy-1,4-naphthoquinone is the most stable form followed by 4-hydroxy-1,2-naphthoquinone, but they are probably in balance with the other tautomeric forms. ${ }^{39}$ This stability of the 1,4-naphthoquinone tautomer is correlated with the dipole moments of the carbonyl groups in combination with intramolecular hydrogen bonds.
3,3'-(Methylene)bis-2-hydroxy-1,4-naphthoquinones 15a-i display cytotoxic effects in $\mathrm{PC} 3$ prostate cancer cells

It has been reported that naphthoquinones with a hydroxyl group at the aromatic ring are more pro-oxidant ${ }^{40}$ and could change their potential anticancer activity. ${ }^{38}$ To test the potential application of 1,4-naphthoquinone derivatives 15a-i as putative anti-cancer agents, their cytotoxic effects were preliminary tested in vitro against the PC3 metastatic human prostate cancer cell line using 3-(4,5-dimethylthiazol-2-yl)-2,5-diphenyltetrazolium bromide (MTT) cell viability assays. Initially, PC3 cells were treated with $100 \mu \mathrm{M}$ of $\mathbf{1 5 a}$-i derivatives. Cell viability was tested 24, 48 and $72 \mathrm{~h}$ posttreatment with the distinct derivative compounds. At $24 \mathrm{~h}$ posttreatment, none of these compounds promoted inhibition of cell viability. Otherwise, one out of these nine tested compounds, named (15c), significantly promoted at least a 55\% decrease of cell viability $(p=0.0397)$. It is noteworthy that compound $\mathbf{1 5 f}$ also promoted a $30 \%$ decrease in PC 3 cell viability, but it did not reach statistical significance $(P=0.201)$. Notably, at $72 \mathrm{~h}$ posttreatment, six out these nine tested compounds significantly inhibited PC 3 cell viability, but the compounds 15c, 15f and 15h $(p<0.05)$ were the most cytotoxic. The other two compounds (15g and 15i) also promoted approximately $30 \%$ inhibition in PC3 cell viability, but that did not reach statistical significance $(p>0.05)$. Compounds without the aryl groups (e.g., 15a and 15c) were not significantly cytotoxic (Figure 4).

\section{Experimental}

\section{General remarks}

Reagents were purchased from Sigma Aldrich, São Paulo, Brazil and were used without further purification. Column chromatography was performed with silica gel 60 (Merck, 70-230 mesh). Analytical thin-layer 


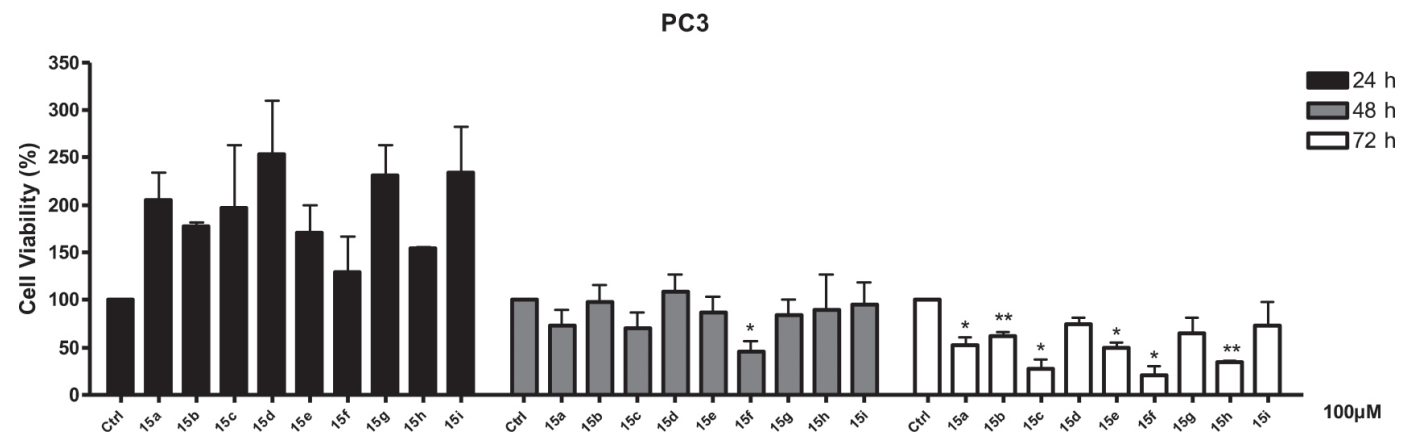

Figure 4. Cell viability of PC3 metastatic prostate cancer cells lines in response to novel 15a-i 1,4-naphthoquinone derivatives. MTT assays using PC3 prostate cancer cell lines have been performed using $100 \mu \mathrm{M}$ of each compound, which were named as shown in Table 1. The results are represented as the mean \pm standard deviation (SD). Statistical analysis was performed using $t$-test $(* p<0.01, * * p<0.001)$.

chromatography (TLC) was performed with silica gel plates (Merck, TLC silica gel 60 F254), and the plots were visualized using UV light or aqueous solutions of ammonium sulfate. Yields refer to chromatographically and spectroscopically homogeneous materials. Melting points were obtained on a Fischer-Johns apparatus and were uncorrected. Infrared spectra were measured using $\mathrm{KBr}$ pellets on a PerkinElmer model 1420 FT-IR Spectrophotometer calibrated relative to the $1601.8 \mathrm{~cm}^{-1}$ absorbance of polystyrene. NMR spectra were recorded on a Varian Unity Plus VXR (500 MHz) instrument in dimethylsulfoxide DMSO- $d_{6}$ and $\mathrm{CDCl}_{3}$ solutions. The chemical shift data were reported in units of $\delta$ (ppm) downfield from tetramethylsilane or the solvent, which were used as an internal standard. Coupling constants $(J)$ are reported in hertz and refer to apparent peak multiplicities. The high resolution mass spectra (electrospray ionization) were obtained using a QTOF Micro (Waters, Manchester, UK) mass spectrometer (HRESIMS). Compounds $\mathbf{1 3 a}^{36}$ and $\mathbf{1 3 d} \mathbf{d}^{38}$ were previously reported, and compounds $\mathbf{1 3 b}$-c and $\mathbf{1 3 e}$-f were prepared by the same method.

\section{General procedures for preparing compounds 13b-c, e-i}

Compounds 13b-c, e-i were synthesized according to the general procedure described in the literature ${ }^{34}$ with modifications. They were obtained by reacting a suspension of lawsone (10 mmol, $1.75 \mathrm{~g}$ ), in $20 \mathrm{~mL}$ of ethanol, with the respective amine $(11 \mathrm{mmol})$. After formation of the lawsonate solution, the aldehyde $(11 \mathrm{mmol})$ is added and the mixture, left stirring at room temperature for $12 \mathrm{~h}$ in the dark. The solids were filtered, washed with ethanol, water, diethyl eter and dried under vacuum.

2-((Benzylamino)( $p$-tolyl)methyl)-3-hydroxynaphthalene1,4-dione (13b)

Orange solid; yield 78\%; mp 173-175 ${ }^{\circ} \mathrm{C}$; IR (KBr) $\mathrm{v} / \mathrm{cm}^{-1} 3076,2979,2720,1679,1578 ;{ }^{1} \mathrm{H}$ NMR
(500.00 MHz, DMSO-d $)_{6} \delta 7.92(\mathrm{dd}, 1 \mathrm{H}, J$ 7.6, $0.8 \mathrm{~Hz})$, 7.84 (dd, 1H, J 7.6, 0.8 Hz), 7.69 (td, 1H, J 7.5, $1.3 \mathrm{~Hz})$, 7.58 (td, 1H, J 7.5, 1.3 Hz), 7.42 (d, 2H, J 8.0 Hz), 7.41-7.37 $(\mathrm{m}, 5 \mathrm{H}), 7.14(\mathrm{~d}, 2 \mathrm{H}, J 8.0 \mathrm{~Hz}), 5.48(\mathrm{~s}, 1 \mathrm{H}), 4.12(\mathrm{~d}, 2 \mathrm{H}$, $J 2.5 \mathrm{~Hz}), 2.26$ (s, 3H); ${ }^{13} \mathrm{C}$ NMR $\left(75.0 \mathrm{MHz}\right.$, DMSO- $\left.d_{6}\right)$ $\delta 193.37,192.75,185.75,185.71,161.97,144.45,140.07$, $138.15,134.29,133.72,131.69,130.10,129.93,129.21$, 129.04, 128.72, 128.40, 128.29, 127.57, 127.39, 120.27, 64.31, 43.06, 20.93; HRMS (FTMS + pESI) $m / z, \mathrm{C}_{25} \mathrm{H}_{22} \mathrm{NO}_{3}$ $[\mathrm{M}]^{+}$observed: 384.1578 , requires: 384.1594 .

2-((Benzylamino)(4-bromophenyl)methyl)-3-hydroxynaphthalene-1,4-dione (13c)

Orange solid; yield 54\%; mp 166-168 ${ }^{\circ} \mathrm{C}$; IR (KBr) $\mathrm{v} / \mathrm{cm}^{-1} 1694,1615,1669,1588,1530 ;{ }^{1} \mathrm{H}$ NMR $\left(500.00 \mathrm{MHz}, \mathrm{DMSO}-d_{6}\right) \delta 7.92(\mathrm{~d}, 1 \mathrm{H}, J 7.6 \mathrm{~Hz}), 7.84$ $(\mathrm{d}, 1 \mathrm{H}, J 7.6 \mathrm{~Hz}), 7.70(\mathrm{td}, 1 \mathrm{H}, J 7.5,1.2 \mathrm{~Hz}), 7.58(\mathrm{td}, 1 \mathrm{H}$, $J 7.5,1.2 \mathrm{~Hz}), 7.53(\mathrm{~d}, 2 \mathrm{H}, J 8.7 \mathrm{~Hz}), 7.50(\mathrm{~d}, 2 \mathrm{H}, J 8.7 \mathrm{~Hz})$, 7.43-7.36 (m, 5H), $5.51(\mathrm{~s}, 1 \mathrm{H}), 4.14(\mathrm{~s}, 2 \mathrm{H}) ;{ }^{13} \mathrm{C} \mathrm{NMR}$ $\left(126.0 \mathrm{MHz}, \mathrm{DMSO}-d_{6}\right) \delta 184.76,183.25,157.95,134.16$, $133.58,131.99,131.40,131.13,130.87,130.23,129.92$, $129.53,129.12,128.81,128.50,128.34,128.23,127.59$, 125.88, 124.90, 63.57, 42.31; HRMS (FTMS + pESI) $\mathrm{m} / \mathrm{z}$, $\mathrm{C}_{24} \mathrm{H}_{19} \mathrm{BrNO}_{3}[\mathrm{M}]^{+}$observed: 448.0522, requires: 448.0543.

2-(1-(Benzylamino)ethyl)-3-hydroxynaphthalene-1,4-dione (13e)

Orange solid; yield 71\%; mp 179-181 ${ }^{\circ} \mathrm{C}$; IR (KBr) $\mathrm{v} / \mathrm{cm}^{-1} 2979,2637,2418,1668,1611,1590,1531 ;{ }^{1} \mathrm{H}$ NMR (500.00 MHz, DMSO- $d_{6}$ ) $\delta 7.92$ (ddd, 1H, J7.6, 1.2, $0.5 \mathrm{~Hz}$ ), 7.82 (ddd, 1H, J 7.6, 1.2, 0.5 Hz), 7.68 (td, 1H, J 7.5 and $1.4 \mathrm{~Hz}), 7.56(\mathrm{td}, 1 \mathrm{H}, J 7.5$ and $1.4 \mathrm{~Hz}), 7.39-7.30(\mathrm{~m}, 5 \mathrm{H})$, 4.51 (q, 1H, J $6.8 \mathrm{~Hz}), 4.01$ (d, 1H, J 13.3 Hz), 3.97 (d, 1H, $J 13.3 \mathrm{~Hz}), 1.41$ (d, 3H, J 6.8 Hz); ${ }^{13} \mathrm{C} \mathrm{NMR}(125.0 \mathrm{MHz}$, DMSO- $\left.d_{6}\right) \delta 184.19,178.60,170.46,134.68,133.48,132.53$, 131.52, 130.62, 129.57, 128.52, 128.43, 125.18, 124.92, 111.26, 51.52, 48.32, 17.62; HRMS (FTMS + pESI) $\mathrm{m} / \mathrm{z}$, $\mathrm{C}_{19} \mathrm{H}_{18} \mathrm{NO}_{3}[\mathrm{M}]^{+}$observed: 308.1291 , requires: 308.1281 . 
2-((Benzylamino)(4-chlorophenyl)methyl)-3-hydroxynaphthalene-1,4-dione (13f)

Orange solid; yield 71\%; mp 147-149 ${ }^{\circ} \mathrm{C}$; IR (KBr) $v / \mathrm{cm}^{-1} 2726,1701,1676,1561,1576,1589 ;{ }^{1} \mathrm{H}$ NMR $\left(500.00 \mathrm{MHz}, \mathrm{DMSO}-d_{6}\right) \delta 7.92(\mathrm{dd}, 1 \mathrm{H}, J 7.6,1.0 \mathrm{~Hz})$, 7.84 (dd, 1H, J 7.6, 1.0 Hz), $7.70(\mathrm{ttd}, 2 \mathrm{H}, J$ 20.0, 7.6, $1.0 \mathrm{~Hz}), 7.57$ (d, 2H, J 8.6 Hz), 7.42-7.36 (m, 5H), 7.34 $(\mathrm{d}, 2 \mathrm{H}, J 8.6 \mathrm{~Hz}), 5.53(\mathrm{~s}, 1 \mathrm{H}), 4.14(\mathrm{~s}, 2 \mathrm{H}) ;{ }^{13} \mathrm{C} \mathrm{NMR}$ $\left(75.0 \mathrm{MHz}, \mathrm{DMSO}-d_{6}\right) \delta 192.62,191.50,160.21,139.71$, $134.90,134.11,133.60,131.14,130.75,129.26,129.08$, 128.76, 128.46, 128.30, 128.19, 127.55, 127.26, 126.97, 63.53, 42.31; HRMS (FTMS + pESI) $m / z, \mathrm{C}_{24} \mathrm{H}_{19} \mathrm{ClNO}_{3}$ $[\mathrm{M}]^{+}$observed: 404.1042; requires: 404.1048.

2-((Benzylamino)(4-fluorophenyl)methyl)-3-hydroxynaphthalene-1,4-dione (13g)

Red solid; yield 63\%; mp 150-152 ${ }^{\circ} \mathrm{C}$; IR (KBr) $\mathrm{v} / \mathrm{cm}^{-1} 3456,2596,2386,1681,1591,1504 ;{ }^{1} \mathrm{H}$ NMR $\left(500.00 \mathrm{MHz}, \mathrm{DMSO}-d_{6}\right) \delta 7.92(\mathrm{~d}, 1 \mathrm{H}, J 7.6 \mathrm{~Hz}), 7.84$ (d, $1 \mathrm{H}, J 7.6 \mathrm{~Hz}), 7.70$ (ttd, 2H, J 21.1, 7.6, 1.3 Hz), 7.58 (dd, $2 \mathrm{H} \mathrm{J} \mathrm{7.7,} \mathrm{6.9} \mathrm{Hz),} \mathrm{7.43-7.36} \mathrm{(m,} \mathrm{5H),} 7.15$ (dd, 1H, J 7.7, $6.9 \mathrm{~Hz}), 5.53(\mathrm{~s}, 1 \mathrm{H}), 4.13(\mathrm{~s}, 2 \mathrm{H}) ;{ }^{13} \mathrm{C} \mathrm{NMR}(75.0 \mathrm{MHz}$, DMSO- $\left.d_{6}\right) \delta 192.65,191.08,160.11,143.63,139.11$, $134.13,133.54,133.43,132.04,131.91,130.95,130.74$, $130.35,129.91,129.80,129.09,128.78,128.47,128.33$, $128.01,126.43,125.08,123.99,122.18,119.98,116.14$, 115.84, 115.44, 115.16, 113.74, 113.46, 63.50, 42.31; HRMS (FTMS + pESI) $m / z, \mathrm{C}_{24} \mathrm{H}_{19} \mathrm{FNO}_{3}[\mathrm{M}]^{+}$observed: 388.1328 , requires: 388.1343 .

2-((Benzylamino)(4-methoxyphenyl)methyl)-3-hydroxynaphthalene-1,4-dione (13h)

Red solid; yield: 84\%; mp 142-144 ${ }^{\circ} \mathrm{C}$; IR (KBr) $\mathrm{v} / \mathrm{cm}^{-1}$ 2948, 2608, 2515, 2359, 1671, 1590; ${ }^{1} \mathrm{H}$ NMR $\left(500.00 \mathrm{MHz}, \mathrm{DMSO}-d_{6}\right) \delta 7.92(\mathrm{dd}, 1 \mathrm{H} J$ 7.6, $1.1 \mathrm{~Hz})$, 7.84 (dd, 1H, J 7.6, 1.1 Hz), 7.69 (td, 1H, J 7.5, 1.3 Hz), $7.58(\mathrm{td}, 1 \mathrm{H}, J 7.5,1.3 \mathrm{~Hz}), 7.46$ (d, 2H, J 8.8 Hz), 7.42-7.36 $(\mathrm{m}, 5 \mathrm{H}), 6.89$ (d, 2H, J $8.8 \mathrm{~Hz}), 5.47(\mathrm{~s}, 1 \mathrm{H}), 4.11(\mathrm{~d}, 2 \mathrm{H}$, $J 2.1 \mathrm{~Hz}), 3.72$ (s, 3H); ${ }^{13} \mathrm{C}$ NMR (125.0 MHz, DMSO- $d_{6}$ ) $\delta 183.45,182.01,156.51,139.29,133.27,132.31,131.38$, $130.82,129.30,128.45,128.28,127.95,127.63,125.39$, $125.00,124.69,122.50,122.15,120.79,119.92,63.62$, 54.71, 42.31; HRMS (FTMS + pESI) $m / z, \mathrm{C}_{25} \mathrm{H}_{22} \mathrm{NO}_{4}[\mathrm{M}]^{+}$ observed: 400.1536, requires: 400.1543.

2-((Benzylamino)(phenyl)methyl)-3-hydroxynaphthalene1,4-dione (13i)

Orange solid; yield 82\%; mp 175-177 ${ }^{\circ} \mathrm{C}$; IR (KBr) $\mathrm{v} / \mathrm{cm}^{-1} 2325,1670,1615,1587,1574,1530 ;{ }^{1} \mathrm{H}$ NMR $\left(500.00 \mathrm{MHz}, \mathrm{DMSO}-d_{6}\right) \delta 7.92(\mathrm{dd}, 1 \mathrm{H}, J 7.6,0.9 \mathrm{~Hz})$, 7.84 (dd, 1H, J 7.6, 0.9 Hz), 7.70 (dt, 1H, J 7.5, 1.3 Hz),
7.58 (dt, 1H, J 7.5, 1.3 Hz), 7.55 (d, 1H, J 1.3 Hz), 7.53 (s, 1H), 7.42-7.36 (m, 5H), 7.33 (dd, 2H, J 8.0, 6.6 Hz, ), 7.28 (dd, 1H, J 8.0, 6.6 Hz), 5.50 (s, 1H), 4.15 (d, 1H, J13.3 Hz), $4.12(\mathrm{~d}, 1 \mathrm{H}, J 13.3 \mathrm{~Hz}) ;{ }^{13} \mathrm{C}$ NMR $\left(125.0 \mathrm{MHz}, \mathrm{DMSO}-d_{6}\right)$ $\delta 184.06,178.63,170.47,138.34,134.46,133.54,132.13$, $131.50,130.76,129.86,128.61,128.39,128.25,127.88$, 127.74, 125.22, 124.97, 110.81, 58.70, 49.03; HRMS (FTMS + pESI) $m / z, \mathrm{C}_{24} \mathrm{H}_{20} \mathrm{NO}_{3}[\mathrm{M}]^{+}$observed: 370.1449 , requires: 370.1438 .

\section{General procedures for preparing compounds 15a-i}

In a round bottom flask, 2,5-dihydroxy1,4-naphthoquinone (1 mmol) and Mannich adduct 13a-h $(0.8 \mathrm{mmol})$ were placed and then dissolved in $15 \mathrm{~mL}$ of acetic acid. The solution was stirred at $100^{\circ} \mathrm{C}$ for $30 \mathrm{~min}$. Then, the reaction mixture was poured into ice-cold distilled water, and the precipitate was filtered under vacuum and washed with distilled water. The brown solid was recrystallized using a pentane/chloroform mixture (9:1) or separated by column chromatography on silica using gradient of hexane an ethyl acetate. The yields obtained for products $\mathbf{1 5 a - i}$ by both purification methods are described in Table 1.

2,5-Dihydroxy-3-((3-hydroxy-1,4-dioxo-1,4-dihydronaphthalen-2-I)methyl)naphthalene-1,4-dione (15a)

Mp 241-243 ${ }^{\circ} \mathrm{C}$; IR (KBr) v / $\mathrm{cm}^{-1} 3296,1643,1666$, 1592,$1613 ;{ }^{1} \mathrm{H}$ NMR $\left(500.00 \mathrm{MHz}, \mathrm{DMSO}-d_{6}\right) \delta 8.00-7.95$ (m, 2H), $7.81(\mathrm{td}, 1 \mathrm{H}, J$ 7.6, 1.4 Hz), $7.77(\mathrm{td}, 1 \mathrm{H}, J$ 7.6, $1.4 \mathrm{~Hz}$ ), 7.63 (ddd, 1H, J 8.4, 7.5, $2.5 \mathrm{~Hz}$ ), 7.52 (ddd, $1 \mathrm{H}, J 7.5,2.5,1.2 \mathrm{~Hz}), 7.28$ (ddd, $1 \mathrm{H}, J 8.4,2.5,1.2 \mathrm{~Hz}$ ), $3.75(\mathrm{~s}, 2 \mathrm{H}) ;{ }^{13} \mathrm{C}$ NMR $\left(125.0 \mathrm{MHz}, \mathrm{DMSO}-d_{6}\right) \delta 190.52$, $190.43,184.09,184.05,181.19,180.62,180.59,160.59$, $157.22,156.79,155.75,155.48,135.66,135.63,134.89$, $133.50,132.60,130.71,130.43,126.34,126.01,125.26$, $122.54,122.10,121.96,121.51,118.88,114.48,18.56$, 17.83, 17.11; HRMS (FTMS + pESI) $m / z, \mathrm{C}_{21} \mathrm{H}_{12} \mathrm{NaO}_{7}$ $[\mathrm{M}]^{+}$observed: 399.046 , requires: 399.0475 .

2,5-Dihydroxy-3-((3-hydroxy-1,4-dioxo-1,4-dihydronaphthalen-2-yl)(p-tolyl)methyl) naphthalene-1,4-dione (15b)

Mp 129-131 ${ }^{\circ} \mathrm{C}$; IR (KBr) v / $\mathrm{cm}^{-1} 3319,2971,1649$, 1619, 1511, 1457; ${ }^{1} \mathrm{H}$ NMR (500.00 MHz, DMSO- $d_{6}$ ) $\delta 7.99(\mathrm{~d}, 1 \mathrm{H}, J 7.2 \mathrm{~Hz}), 7.94(\mathrm{t}, 1 \mathrm{H}, J 7.6 \mathrm{~Hz}), 7.81(\mathrm{t}$, 1H, J 7.6 Hz), 7.79-7.74 (m, 1H), 7.63-7.59 (m, 1H), 7.50 (t, 1H, J 7.4 Hz), $7.26(\mathrm{dd}, 1 \mathrm{H}, J 7.4,2.5 \mathrm{~Hz}), 7.12(\mathrm{~d}, 2 \mathrm{H}$, $J 7.8 \mathrm{~Hz}), 7.00$ (d, 2H, J 7.8 Hz), 6.03 (s, 1H), 2.25 (s, $3 \mathrm{H}) ;{ }^{13} \mathrm{C}$ NMR $\left(125.0 \mathrm{MHz}, \mathrm{DMSO}-d_{6}\right) \delta 189.94,183.92$, $183.83,182.17,182.11,181.96,181.84,160.62,160.56$, 
$157.25,138.21,138.11,137.90,134.99,134.92,134.77$ $134.68,134.58,134.54,133.34,133.20,132.98,132.92$, $131.31,131.09,130.70,130.48,128.81,128.73,128.66$, $128.45,128.11,127.80,126.44,126.42,125.90,125.86$, $125.02,124.83,123.92,123.75,122.50,122.40,118.45$, 118.35, 115.02, 114.97, 37.56, 35.78, 20.99, 20.97, 20.94; HRMS (FTMS + pESI) $m / z, \mathrm{C}_{28} \mathrm{H}_{18} \mathrm{NaO}_{7}[\mathrm{M}]^{+}$observed: 489.0923, requires: 489.0945 .

3-((4-Bromophenyl)(3-hydroxy-1,4-dioxo-1,4-dihydronaphthalen-2-yl)methyl)-2,5-dihydroxynaphthalene1,4-dione (15c)

Mp 139-141 ${ }^{\circ} \mathrm{C}$; IR (KBr) v / $\mathrm{cm}^{-1} 3331,1649,1618$, 1457, 1368; ${ }^{1} \mathrm{H}$ NMR (500.00 MHz, DMSO-d $\left.d_{6}\right) \delta 7.98$ (ddd, 1H, J 7.6, 2.3, 1.5 Hz), 7.94 (t, 1H, J 7.5 Hz), 7.81 (t, 1H, J 7.5 Hz), 7.77 (ddd, 1H, J 7.6, 2.3, 1.5 Hz), 7.62 (ddd, 1H, J 8.2, 7.6, 4.3 Hz), 7.53-7.49 (m, 1H), 7.36 (dd, $2 \mathrm{H}, J 8.1,1.9 \mathrm{~Hz}$ ), 7.26 (ddd, $1 \mathrm{H}, J 8.2,4.3,0.8 \mathrm{~Hz}$,), 7.21 (d, 2H, $J 8.1 \mathrm{~Hz}$ ), 6.01 (s, 1H); ${ }^{13} \mathrm{C}$ NMR (125.0 MHz, DMSO- $\left.d_{6}\right) \delta 190.14,190.07,183.88,183.82,181.77$, $181.63,181.41,160.79,160.66,160.62,159.61,157.79$, $156.87,141.01,140.83,140.62,135.34,135.22,135.01$, $134.92,133.45,133.38,132.85,132.82,131.11,130.97$, $130.94,130.91,130.89,130.82,130.74,130.58,130.50$, $130.43,126.48,125.97,125.22,125.07,123.26,122.95$, $122.23,121.93,118.92,118.90,118.68,118.60,114.85$, 114.79, 37.84, 36.53, 34.94; HRMS (FTMS + pESI) $\mathrm{m} / z$, $\mathrm{C}_{27} \mathrm{H}_{14} \mathrm{BrO}_{7}[\mathrm{M}]^{-}$observed: 528.9934, requires: 528.9928.

2,5-Dihydroxy-3-((3-hydroxy-1,4-dioxo-1,4-dihydronaphthalen-2-yl)(4-nitrophenyl) methyl)naphthalene1,4-dione (15d)

Mp 155-157 ${ }^{\circ} \mathrm{C}$; IR (KBr) v / $\mathrm{cm}^{-1} 3331,1650,1619$, 1514,$1457 ;{ }^{1} \mathrm{H}$ NMR $\left(500.0 \mathrm{MHz}, \mathrm{DMSO}-d_{6}\right) \delta 8.06(\mathrm{~d}$, $2 \mathrm{H}, J 8.8 \mathrm{~Hz}$ ), 7.99 (ddd, 1H, J 7.6, 3.3, $1.1 \mathrm{~Hz}$ ), 7.95 (ddd, 1H, J 11.4, 7.6, 1.1 Hz), 7.82 (t, 1H, J 7.4 Hz, ), 7.77 (ddd, 1H, J 7.4, 3.3, 1.1 Hz), 7.64-7.59 (m, 1H), 7.56-7.49 $(\mathrm{m}, 3 \mathrm{H}), 7.27(\mathrm{~d}, 1 \mathrm{H}, J 8.8 \mathrm{~Hz}), 6.17(\mathrm{~s}, 1 \mathrm{H}) ;{ }^{13} \mathrm{C} \mathrm{NMR}$ $\left(125.0 \mathrm{MHz}, \mathrm{DMSO}-d_{6}\right) \delta 189.81,183.70,183.68,181.81$, $181.71,181.60,160.68,160.64,150.29,150.15,149.95$, $146.21,135.21,135.12,134.98,134.85,133.43,133.34$, $132.90,132.87,129.75,129.50,129.27,129.18,129.07$ $126.50,126.00,125.97,125.11,124.95,123.28,123.19$, 123.09, 118.64, 118.56, 114.97, 38.16, 36.67; HRMS $\left(\mathrm{FTMS}+\right.$ pESI) $m / z, \mathrm{C}_{27} \mathrm{H}_{14} \mathrm{NO}_{9}{ }^{-}[\mathrm{M}]^{-}$observed: 496.0677, requires: 496.0669 .

2,5-Dihydroxy-3-(1-(3-hydroxy-1,4-dioxo-1,4-dihydronaphthalen-2-yl)ethyl)naphthalene-1,4-dione (15e)

Mp 101-103 ${ }^{\circ} \mathrm{C}$; IR (KBr) v / cm ${ }^{-1} 3330,1648,1617$, 1457,$1365 ;{ }^{1} \mathrm{H}$ NMR $\left(500.00 \mathrm{MHz}, \mathrm{DMSO}-d_{6}\right) \delta 7.97-7.93$ (m, 2H), $7.81(\mathrm{t}, 1 \mathrm{H}, J 7.5 \mathrm{~Hz}), 7.75(\mathrm{td}, 1 \mathrm{H}, J 7.5,1.4 \mathrm{~Hz})$, 7.64-7.58 (m, 1H), 7.52-7.48 (m, 1H), 7.29-7.24 (m, 1H), $4.68(\mathrm{q}, 1 \mathrm{H}, J 7.4 \mathrm{~Hz}), 1.61(\mathrm{~d}, 3 \mathrm{H}, J 7.4 \mathrm{~Hz}) ;{ }^{13} \mathrm{C} \mathrm{NMR}$ $\left(125.0 \mathrm{MHz}, \mathrm{DMSO}-d_{6}\right) \delta 190.76,190.74,190.57,184.26$, $184.17,181.84,181.76,181.33,160.66,160.61,157.74$, $156.20,155.57,135.41,135.29,134.97,134.93,133.60$, $133.37,133.33,132.84,130.59,130.26,130.17,126.39$, $125.99,125.85,125.45,125.29,125.16,125.00,118.62$, $118.54,114.71,114.67,111.56,28.85,27.78,17.58,17.41$, 17.17; HRMS (FTMS + pESI) $m / z, \mathrm{C}_{22} \mathrm{H}_{13} \mathrm{O}_{7}[\mathrm{M}]^{-}$observed: 389.0682, requires: 389.0661 .

3-((4-Chlorophenyl)(3-hydroxy-1,4-dioxo-1,4-dihydronaphthalen-2-yl)methyl)-2,5-dihydroxynaphthalene1,4-dione (15f)

Mp 133-135 ${ }^{\circ} \mathrm{C}$; IR (KBr) v / $\mathrm{cm}^{-1} 3332,1649,1619$, 1490, 1457; ${ }^{1} \mathrm{H}$ NMR (500.00 MHz, DMSO- $\left.d_{6}\right) \delta 7.98$ (ddd, 1H, J 7.5, 3.4, 1.3 Hz), $7.94(\mathrm{td}, 1 \mathrm{H}, J 7.6,1.4 \mathrm{~Hz})$, $7.81(\mathrm{td}, 1 \mathrm{H}, J$ 7.6, $1.4 \mathrm{~Hz}), 7.76(\mathrm{ddd}, 1 \mathrm{H}, J$ 7.5, 3.4, $1.3 \mathrm{~Hz}), 7.63-7.58(\mathrm{~m}, 1 \mathrm{H}), 7.52-7.48(\mathrm{~m}, 1 \mathrm{H}), 7.26(\mathrm{dd}$, $2 \mathrm{H}, J$ 8.4, $1.5 \mathrm{~Hz}), 7.22(\mathrm{dd}, 2 \mathrm{H}, J 8.4,1.5 \mathrm{~Hz}), 6.05$ $(\mathrm{s}, 1 \mathrm{H}) ;{ }^{13} \mathrm{C} \mathrm{NMR}\left(125.00 \mathrm{MHz}, \mathrm{DMSO}-d_{6}\right) \delta 190.11$, 190.05, 183.88, 183.81, 181.81, 181.66, 181.47, 161.00, $160.66,160.62,159.80,157.89,156.92,140.52,140.35$, $140.14,135.30,135.19,135.00,134.91,133.44,133.36$, $132.83,131.14,130.94,130.60,130.54,130.52,130.50$, $130.44,130.29,130.04,129.21,129.08,128.98,128.05$, $127.96,127.89,126.48,125.97,125.95,125.20,125.04$, $123.33,123.03,122.26,121.98,118.66,118.58,114.88$, 114.82, 37.75, 36.38, 34.75; HRMS (FTMS + pESI) $\mathrm{m} / \mathrm{z}$, $\mathrm{C}_{27} \mathrm{H}_{14} \mathrm{ClO}_{7}[\mathrm{M}]^{-}$observed: 485.0452, requires: 485.0428 .

3-((4-Fluorophenyl)(3-hydroxy-1,4-dioxo-1,4-dihydronaphthalen-2-yl)methyl)-2,5-dihydroxynaphthalene1,4-dione (15g)

Mp 122-125 ${ }^{\circ} \mathrm{C}$; IR (KBr) v / $\mathrm{cm}^{-1} 3665,3338,2972$, 1649, 1619, 1508; ${ }^{1} \mathrm{H}$ NMR (500.00 MHz, DMSO- $\left.d_{6}\right)$ $\delta 7.99(\mathrm{dt}, 1 \mathrm{H}, J 7.6,1.6 \mathrm{~Hz}), 7.94(\mathrm{td}, 1 \mathrm{H}, J 7.5,1.2 \mathrm{~Hz})$, $7.82(\mathrm{td}, 1 \mathrm{H}, J 7.5,1.2 \mathrm{~Hz}), 7.79-7.75(\mathrm{~m}, 1 \mathrm{H}), 7.68-7.57$ (m, 1H), 7.51 (ddd, 1H, J 7.3, 5.2, 1.0 Hz), 7.33-7.23 (m, $3 \mathrm{H}), 6.99(\mathrm{td}, 2 \mathrm{H}, J$ 8.9, $2.0 \mathrm{~Hz}), 6.03(\mathrm{~s}, 1 \mathrm{H}) ;{ }^{13} \mathrm{C} \mathrm{NMR}$ $\left(125.00 \mathrm{MHz}, \mathrm{DMSO}-d_{6}\right) \delta 190.18,190.11,183.94,183.88$, $181.85,181.70,181.49,162.59,160.84,160.65,160.61$, $159.62,159.39,157.76,156.80,133.44,133.37,132.86$, $132.83,131.10,130.90,130.56,130.43,130.32,130.21$, $130.11,129.97,129.86,126.47,125.95,125.21,125.06$, $123.67,123.36,122.61,122.31,118.66,118.58,114.86$, $114.78,114.71,114.58,114.50,114.43,37.63,36.29$, 34.67; HRMS (FTMS + pESI) $m / z, \mathrm{C}_{27} \mathrm{H}_{15} \mathrm{FNaO}_{7}[\mathrm{M}]^{+}$ observed: 493.0679, requires: 493.0694. 
2,5-dihydroxy-3-((3-hydroxy-1,4-dioxo-1,4-dihydronaphthalen-2-yl)(4-methoxyphenyl) methyl)naphthalene1,4-dione (15h)

Mp 229-231 ${ }^{\circ} \mathrm{C}$; IR (KBr) v / $\mathrm{cm}^{-1} 3233,1642,1615$, 1594; ${ }^{1} \mathrm{H}$ NMR $\left(500.00 \mathrm{MHz}, \mathrm{DMSO}-d_{6}\right) \delta 7.98(\mathrm{dd}, 1 \mathrm{H}$, $J$ 7.5, $1.2 \mathrm{~Hz}), 7.93(\mathrm{td}, 1 \mathrm{H}, J$ 7.6, $1.3 \mathrm{~Hz}), 7.81(\mathrm{td}, 1 \mathrm{H}$, $J$ 7.6, 1.3 Hz), 7.77 (td, $1 \mathrm{H}, J 7.5,1.2 \mathrm{~Hz}), 7.62$ (ddd, 2H, $J$ 8.3, 7.5, 4.5 Hz), 7.51 (ddd, $1 \mathrm{H}, J$ 7.5, 4.5, 1.1 Hz), 7.27 (ddd, $1 \mathrm{H}, J 8.3,4.5,1.1 \mathrm{~Hz}), 7.15$ (d, 2H, $J 8.7 \mathrm{~Hz}), 6.77$ (dd, 2H, J 8.7, 1.8 Hz), 5.97 (s, 1H), $3.71(\mathrm{~s}, 3 \mathrm{H}) ;{ }^{13} \mathrm{C}$ NMR $\left(125.00 \mathrm{MHz}, \mathrm{DMSO}-d_{6}\right) \delta 190.36,190.27,184.05,183.99$, 181.82 , 181.69, 181.64, 181.42, 160.66, 160.63, 160.22, $158.97,157.87,157.85,157.33,156.49,135.35,135.23$, 134.99, 134.92, 133.43, 133.36, 132.94, 132.87, 132.85, $132.75,131.02,130.83,130.50,130.39,129.69,129.51$, $129.27,126.46,125.93,125.24,125.10,124.19,123.83$, $123.22,122.83,118.64,118.57,114.83,114.77,113.78$, 113.71, 113.66, 55.48, 37.62, 36.43, 34.89; HRMS (FTMS + pESI) $m / z, \mathrm{C}_{28} \mathrm{H}_{17} \mathrm{O}_{8}[\mathrm{M}]^{-}$observed: 481.0947, requires: 481.0923.

2,5-Dihydroxy-3-((3-hydroxy-1,4-dioxo-1,4-dihydronaphthalen-2-yl)(phenyl)methyl) naphthalene-1,4-dione (15i)

Mp 117-119 ${ }^{\circ} \mathrm{C}$; IR (KBr) v / cm ${ }^{-1} 3332,2969,1649$, 1620,$1457 ;{ }^{1} \mathrm{H}$ NMR $\left(500.00 \mathrm{MHz}\right.$, DMSO- $\left.d_{6}\right) \delta 8.03-7.91$ $(\mathrm{m}, 2 \mathrm{H}), 7.86-7.73(\mathrm{~m}, 2 \mathrm{H}), 7.62(\mathrm{td}, 1 \mathrm{H}, J 7.7,2.2 \mathrm{~Hz})$, 7.52 (dd, 1H, J 7.2, 3.0 Hz), 7.32-7.19 (m, 5H), 7.19-7.09 $(\mathrm{m}, 1 \mathrm{H}), 6.10(\mathrm{~s}, 1 \mathrm{H}) ;{ }^{13} \mathrm{C}$ NMR $\left(125.00 \mathrm{MHz}\right.$, DMSO- $\left.d_{6}\right)$ $\delta$ 190.34, 190.26, 184.01, 183.97, 181.78, 181.65, 181.36, $160.68,160.65,160.23,159.07,157.48,156.69,141.24$, $141.10,140.93,135.38,135.26,134.99,134.94,133.44$, $133.38,132.87,132.85,130.85,130.52,130.41,129.21$, $129.08,128.98,128.64,128.46,128.23,128.13,128.06$, $128.01,126.49,125.96,125.89,125.25,125.12,123.81$, 123.44, 122.90, 118.68, 118.61, 114.83, 114.78, 38.27, 37.13, 35.66; HRMS (FTMS + pESI) $m / z, \mathrm{C}_{27} \mathrm{H}_{15} \mathrm{O}_{7}^{-}[\mathrm{M}]^{-}$ observed: 451.0830 , requires: 451.0818 .

\section{Methylation of $15 \mathrm{i}$ with diazomethane}

${ }^{1} \mathrm{H} \mathrm{NMR}\left(500 \mathrm{MHz}, \mathrm{CDCl}_{3}\right) \delta 12.16(\mathrm{~s}, 1 \mathrm{H}), 8.08-8.03$ (m, 2H), 7.74-7.68 (m, 3H), 7.61-7.54 (m, 2H), 7.33-7.27 $(\mathrm{m}, 5 \mathrm{H}), 7.26-7.20(\mathrm{~m}, 1 \mathrm{H}), 6.08(\mathrm{~s}, 1 \mathrm{H}), 3.77(\mathrm{~s}, 3 \mathrm{H}), 3.74$ (s, 3H), 3.73 (s, 3H); $\left.{ }^{13} \mathrm{C} \mathrm{NMR} \mathrm{(75} \mathrm{MHz,} \mathrm{CDCl}_{3}\right) \delta 190.48$, 184.97, 184.94, 182.11, 182.07, 181.46, 161.36, 159.17, $158.74,158.44,140.47,140.14,135.64,135.43,134.95$, $134.60,134.27,134.17,133.59,133.49,132.20,132.12$, $131.77,131.65,128.66,128.54,128.38,128.30,126.81$, $126.67,126.56,126.34,126.27,125.02,119.16,114.53$, 61.06, 60.94, 40.19, 39.44, 29.90.
Cell lines and culture conditions

In this study, we used the PC3 metastatic prostate cancer cell line, derived from bone metastasis. This cell line was obtained from American Type Cell Culture Collection (ATCC) and consisted of short tandem repeat (STR) fingerprinted to confirm identity. The cell line was cultured in RPMI (RPMI-1640 Medium HEPES Modification R5886, Sigma Aldrich) containing 10\% fetal bovine serum (FBS), $1 \%$ L-glutamine (L-Glu) and grown from $1 \%$ until reaching $80-90 \%$ cell confluence. Cell were incubated at $37^{\circ} \mathrm{C}$ in a $5 \% \mathrm{CO}_{2}$ atmosphere.

Cell treatment with bis-1,4-naphthoquinone derivatives 15a-i

Before PC 3 cell treatment with each one of the 9 tested compounds 15a-i, the compounds were reconstituted in dimethyl sulfoxide (DMSO) to maintain their stability at a stock solution of $10 \mathrm{mM}$. These compounds were then diluted in phosphate buffered saline (PBS) to prepare a working solution at $1 \mathrm{mM}$. A final concentration of $100 \mathrm{mM}$ has been used for each compound. PC 3 cells were plated in culture plates containing 96 wells, and $24 \mathrm{~h}$ after plating, cells were treated with $100 \mu \mathrm{M}$ of each compound 15a-i. Control cells were treated with DMSO/PBS solution, which was used at each time point to calculate relative cell viability as $100 \%$ of cell viability.

\section{MTT assays}

The 3-(4,5-dimethylthiazol-2-yl)-2,5-diphenyltetrazolium bromide (MTT) assay was used to measure the cytotoxic effects of distinct tested naphthoquinone derivatives 15a-i on PC3 cells. Briefly, cells were seeded $\left(1 \times 10^{4}\right.$ cells per well) in 96-well plates. After adhesion, cells were exposed to various concentrations of these compounds for 24, 48 and $72 \mathrm{~h}$. Compound-free medium was added to the control wells. A total of $20 \mu \mathrm{L}$ of MTT ( $5 \mathrm{mg} \mathrm{mL}^{-1}$ ) was added to each well $4 \mathrm{~h}$ before the end of each time point. MTT solution was then removed, and the insoluble formazan crystals were dissolved in $150 \mu \mathrm{L}$ of DMSO (Sigma-Aldrich, MO, USA). The optical density at $570 \mathrm{~nm}$ was determined using Spectra Max 190. All experiments were performed in triplicate. Statistical significance for various parameters was estimated using Student's $t$-test (two-tailed). Alpha level was set to the value of 0.05 for all statistical tests, and value of $p<0.05$ was considered to be statistically significant. Graph Pad Prism was used to plot bar graphs. ${ }^{41}$ 


\section{Conclusions}

A novel method for the synthesis of nonsymmetrical 3,3'-(aryl/alkyl-methylene) bis-2-hydroxy-1,4-naphthoquinones was developed, which was used to prepare compounds 15a-i in moderate to good yields. This new class of compounds had their structures adequately elucidated by spectroscopic techniques. Preliminary screening of these compounds regarding their effect on PC3 metastatic prostate cancer cell viability by MTT assays at $100 \mu \mathrm{M}$ revealed that compounds $\mathbf{1 5 c}, \mathbf{1 5 f}$ and $\mathbf{1 5 h}$ present promising activity in PC3 cells. Further work should be conducted to investigate the scope of this method for producing new 3,3'-(aryl/alkyl-methylene)bis-2-hydroxy1,4-naphthoquinones followed by assays in PC 3 metastatic prostate cancer cells to investigate the mechanism by which they promote PC3 metastatic prostate cell cytotoxicity.

\section{Supplementary Information}

Supplementary data are available free of charge at http://jbcs.sbq.org.br as PDF file.

\section{Acknowledgments}

Fellowships granted by CNPq, CAPES and FAPERJ are gratefully acknowledged. This work was partially supported by FAPERJ grant No. E-26/201.448/2014 and CNPq 303713/2014-3. The also author thanks FIOCRUZ for HRESIMS. We also kindly thank FAPERJ, grant No. E-26/111.227/2014; E-26/010.002007/2014; E-26/203.204/2015; CNPq grant No.: 310591/2014-7 and 312158/2017-3; FCT/CAPES grant No. 99999.008550/2014-00; and Ministério da Saúde and UFF/PROPPI.

\section{References}

1. Ferreira, D. C. M.; Tapsoba, I.; Arbault, S.; Bouret, Y.; Moreira, M. S. A.; Pinto, A. V.; Goulart, M. O. F.; Amatore, C.; ChemBioChem 2008, 10, 528.

2. Izumi, E.; Ueda-Nakamura, T.; Dias Filho, B. P.; Veiga Jr., V. F.; Nakamura, C. V.; Nat. Prod. Rep. 2011, 28, 809.

3. Salmon-Chemin, E.; Buisine, V.; Yardley, S.; Kohler, M. A.; Debreu, V.; Landry, C.; Sergheraert, S. L.; Croft, R. L.; Krauth-Siegel, R. L.; Davioud-Charvet, E.; J. Med. Chem. 2001, 44,548 .

4. Müller, L.; Johann, B.; Jannack, M.; Brückner, D. A.; Lanfranchi, H.; Bauer, C.; Sanchez, V.; Yardley, C.; Deregnaucourt, J.; Schrevel, M.; Lanzer, R. H.; Schirmer, R. H.; Davioud-Charvet, E.; J. Am. Chem. Soc. 2011, 133, 11557.
5. Santos, V. M.; Carneiro, J. W. M.; Ferreira, V. F.; Bioorg. Med. Chem. 2004, 12, 87.

6. Hickman, A.; Curr. Opin. Genet. Dev. 2002, 12, 67.

7. Zani, L.; Chiari, E.; Krettli, A. U.; Murta, S. M. F.; Cunningham, M. L.; Fairlamb, A. H.; Romanha, A. J.; Bioorg. Med. Chem. 1997, 5, 2185.

8. Villamil, S. F.; Stoppani, A. O. M.; Dubin, M.; Methods Enzymol. 2004, 378, 67.

9. Gopinath, P.; Mahammed, A.; Ohayon, S.; Gross, Z.; Brik, A.; Chem. Sci. 2016, 7, 7079.

10. Canfield, C. J.; Pudney, M.; Gutteridge, W. E.; Exp. Parasitol. 1993, 80, 373.

11. Hashemi-Fesharki, R.; Res. Vet. Sci. 1991, 50, 204.

12. https://www.cancer.gov/about-cancer/treatment/clinical-trials/ search/v?id=NCI-2015-01450\&r=1, accessed in June 2019.

13. Forezi, L. S. M.; Marra, R. K. F.; da Silva, F. C.; Ferreira, V. F.; Curr. Org. Synth. 2017, 14, 929.

14. Casiraghi, G.; Casnati, G.; Pochini, A.; Ungaro, R.; J. Chem. Soc. 1982, 1, 805.

15. Casiraghi, G.; Casnati, G.; Cornia, M.; Sartori, G.; Ungaro, R.; J. Chem. Soc. 1974, 1, 2077.

16. Bennett, D. J.; Dean, F. M.; Herbin, G. A.; Matkin, D. A.; Price, A. W.; J . Chem. Soc. 1980, 1, 1978.

17. Jung, J. C.; Oh, S.; Molecules 2011, 17, 240.

18. Su, C. X.; Mouscadet, J. F.; Chiang, C. C.; Tsai, H. J.; Hsu, L.Y.; Chem. Pharm. Bull. 2006, 54, 682.

19. Hayupakaranant, P.; Noguchi, H.; De-Eknamkul, W.; Sankawa, U.; Phytochemistry 1995, 40, 1141.

20. Tisseh, Z. N.; Bazgir, A.; Dyes Pigm. 2009, 83, 258.

21. de Oliveira, A. S.; Lianes, L. C.; Nunes, R. J.; Yunes, R. A.; Brighente, I. M. C.; Green Sustainable Chem. 2014, 4, 177.

22. de Araújo, M. V.; de Souza, P. S. O.; de Queiroz, A. C.; da Matta, C. B. B.; Leite, A. B.; da Silva, A. E.; de França, J. A. A.; Silva, T. M. S.; Camara, C. A.; Alexandre-Moreira, M. S.; Molecules 2014, 19, 15180

23. Sharma, G.; Chowdhury, S.; Sinha, S.; Majumder, H. K.; Kumar, S. V.; J. Enzyme Inhib. Med. Chem. 2014, 29, 185.

24. Sadhukhan, P.; Saha, S.; Sinha, K.; Brahmachari, G.; Sil, P. C.; PLoS One 2016, 11, 158694.

25. Brahmachari, G.; ACS Sustainable Chem. Eng. 2015, 3, 2066.

26. Jardim, G. A. M.; Cruz, E. H. G.; Valença, W. O.; Lima, D. J. B.; Cavalcanti, B. C.; Pessoa, C.; Rafique, J.; Braga, A. L.; Jacob, C.; Silva Jr., E. N.; Molecules 2018, 23, 83.

27. Costa, M. P.; Feitosa, A. C. S.; Oliveira, F. C. E.; Cavalcanti, B. C.; da Silva, E. N.; Dias, G. G.; Sales, F. A. M.; Sousa, B. L.; Barroso-Neto, I. L.; Pessoa, C.; Caetano, E. W. S.; Di Fiore, S.; Fischer, R.; Ladeira, L. O.; Freire, V. N.; Molecules 2016, 21, 873.

28. Huang, H.; Xie, H.; Pan, Y.; Zheng, K.; Xia, Y.; Chen, W.; Cell Physiol. Biochem. 2018, 45, 267. 
29. Hafeez, B. B.; Zhong, W.; Fischer, J. W.; Mustafa, A.; Shi, X.; Meske, L.; Hong, H.; Cai, W.; Havighurst, T.; Kim, K.; Verma, A. K.; Mol. Oncol. 2013, 7, 428.

30. Gara, R. K.; Srivastava, V. K.; Duggal, S.; Bagga, J. K.; Bhatt, M.; Sanyal, S.; Mishra, D. P.; J. Biomed. Sci. 2015, 22, 26.

31. Prasad, C. V.; Nayak, V. L.; Ramakrishna, S.; Mallavadhani, U. V.; Chem. Biol. Drug Des. 2018, 91, 220.

32. Pidugu, L. S. M.; Mbimba, J. C. E.; Ahmad, M.; Pozharski, E.; Sausville, E. A.; Emadi, A.; Toth, E. A.; BMC Struct. Biol. 2016, 16,1 .

33. Ross, A. E.; Emadi, A.; Marchionni, L.; Hurley, P. J.; Simons, B. W.; Schaeffer, E. M.; Vuica-Ross, M.; BJU Int. 2011, 108, 447.

34. Leffler, T.; Hathaway, R. J.; J. Am. Chem. Soc. 1948, 70, 322.

35. Silva, G. B.; Neves, A. P.; Vargas, M. D.; Marinho-Filho, J. D. B.; Costa-Lotufo, L. V.; Bioorg. Med. Chem. Lett. 2016, $26,3537$.
36. Neves, A. P.; Barbosa, C. C.; Greco, S. J.; Vargas, M. D.; Visentin, L. C.; Pinheiro, C. B.; Mangrich, A. S.; Barbosa, J. P.; da Costa, G. L.; J. Braz. Chem. Soc. 2009, 20, 712.

37. Ghodsi, R.; Safarnejad, M.; Malayeri, S. O.; Golmakanion, S.; J. Chem. Res. 2018, 42, 224.

38. da Rocha, D. R.; de Souza, A. C.; Resende, J. A.; Santos, W. C.; dos Santos, E. A.; Pessoa, C.; de Moraes, M. O.; Costa-Lotufo, L. V.; Montenegro, R. C.; Ferreira, V. F.; Org. Biomol. Chem. 2011, 9, 4315.

39. Lamoureux, G.; Perez, A.; Araya, M.; Agüero, C.; J. Phys. Org. Chem. 2008, 21, 1022.

40. Murakami, K.; Haneda, M.; Iwata, S.; Yoshino, M.; Toxicol In Vitro 2010, 24, 905.

41. GraphPad Prism, version 8; GraphPad Software Inc., San Diego, USA, 2018.

Submitted: February 5, 2019

Published online: July 24, 2019 